International Journal of Modern Physics B (C) World Scientific Publishing Company

\title{
What a Two Solar Mass Neutron Star Really Means
}

\author{
JAMES M. LATTIMER \\ Department of Physics and Astronomy \\ Stae University of New York at Stony Brook \\ Stony Brook, NY 11794-3800, USA \\ lattimer@mail.astro.sunysb.edu \\ MADAPPA PRAKASH \\ Department of Physics and Astronomy, Ohio University \\ Athens, Ohio 45701, USA \\ prakash@harsha.phy.ohiou.edu \\ Received Day Month Year \\ Revised Day Month Year
}

\begin{abstract}
The determination of neutron star masses is reviewed in light of a new measurement of $1.97 \mathrm{M}_{\odot}$ for PSR J1614-2230 and an estimate of $2.4 \mathrm{M}_{\odot}$ for the black widow pulsar. Using a simple analytic model related to the so-called maximally compact equation of state, model-independent upper limits to thermodynamic properties in neutron stars, such as energy density, pressure, baryon number density and chemical potential, are established which depend upon the neutron star maximum mass. Using the largest well-measured neutron star mass, $1.97 \mathrm{M}_{\odot}$, it is possible to show that the energy density can never exceed about $2 \mathrm{GeV}$, the pressure about $1.3 \mathrm{GeV}$, and the baryon chemical potential about $2.1 \mathrm{GeV}$. Further, if quark matter comprises a significant component of neutron star cores, these limits are reduced to $1.3 \mathrm{GeV}, 0.9 \mathrm{GeV}$, and $1.5 \mathrm{GeV}$, respectively. We also find the maximum binding energy of any neutron star is about $25 \%$ of the rest mass. Neutron matter properties and astrophysical constraints additionally imply an upper limit to the neutron star maximum mass of about $2.4 \mathrm{M}_{\odot}$. A measured mass of $2.4 \mathrm{M}_{\odot}$ would be incompatible with hybrid star models containing significant proportions of exotica in the form of hyperons, Bose condensates or quark matter.
\end{abstract}

Keywords: neutron star masses; equation of state.

\section{Introduction}

One of the most fascinating stories in astrophysics concerns the accumulation of precisely measured neutron star masses. Gerry has had a long-time interest in these measurements, as for many years he has maintained that the neutron star maximum mass is no greater than (1.5-1.6) $\mathrm{M}_{\odot}$. From a theoretical perspective, a maximum mass of $1.5 \mathrm{M}_{\odot}$ fits his fondness for the effects of kaon condensation proposed by Kaplan and Nelson 1, the effective kaon mass falls with increasing density and the eventual onset of kaon condensation at a few times the nuclear saturation density softens the equation of state (hereafter, EOS) and leads to a rather small maximum 
mass. He takes as observational support of this thesis the "missing" neutron star in the remnant of SN 1987A, which had a baryon mass not in excess of $1.7 \mathrm{M}_{\odot}$ based on estimates of the initial mass of the star and its ejected mass 2 . He reasons that the maximum stable mass of the proto-neutron star as it deleptonized must have eventually fallen below its actual mass, prompting a collapse to a black hole ${ }^{3}$. Of course, this had to have happened more than 12 seconds after the core collapse since neutrinos were observed during that time period and their emission is believed to abruptly cease 4 once the black hole's event horizon forms.

\section{Measurements of Neutron Star Masses}

The most accurate measurements of neutron star masses are for pulsars in bound binary systems. In these systems, five Keplerian parameters can be precisely measured by pulse-timing techniques 5 , including the binary period $P$, the projection of the pulsar's semimajor axis on the line of sight $a_{p} \sin i$ (where $i$ is the binary inclination angle), the eccentricity $e$, and the time and longitude of periastron $T_{0}$ and $\omega$. Combining two of the observational parameters, one can form the mass function:

$$
f_{p}=\left(\frac{2 \pi}{P}\right)^{2}\left(\frac{a_{p} \sin i}{c}\right)^{3} \frac{\mathrm{M}_{\odot}}{\mathrm{T}_{\odot}}=\frac{\left(M_{c} \sin i\right)^{3}}{M^{2}} \mathrm{M}_{\odot},
$$

where $M=M_{p}+M_{c}$ is the total mass, $M_{p}$ is the pulsar mass, and $M_{c}$ is the companion mass (all measured in $\mathrm{M}_{\odot}$ units). The constant $\mathrm{T}_{\odot}=G \mathrm{M}_{\odot} / c^{3}$ is 4.9255 $\mu \mathrm{s}$. The mass function $f_{p}$ is also equal to the minimum possible mass $M_{c}$ for the companion.

The inclination angle $i$ is often the most difficult aspect to infer, but even if it was known a priori the above equation only specifies a relation between $M_{p}$ and $M_{c}$ unless the mass function $f_{c}$ of the companion is also measurable. This occurs in the rare case when the companion is itself a pulsar or a star with an observable spectrum.

Fortunately, binary pulsars are compact systems and general relativistic effects can often be observed. These include the advance of the periastron of the orbit

$$
\dot{\omega}=3\left(\frac{2 \pi}{P}\right)^{5 / 3}\left(M \mathrm{~T}_{\odot}\right)^{2 / 3}\left(1-e^{2}\right)^{-1},
$$

the combined effect of variations in the tranverse Doppler shift and gravitational redshift around an elliptical orbit

$$
\gamma=e\left(\frac{P}{2 \pi}\right)^{1 / 3} \frac{M_{c}\left(M+M_{c}\right)}{M^{4 / 3}} \mathrm{~T}_{\odot}^{2 / 3}
$$

and the orbital period decay due to the emission of gravitational radiation

$$
\dot{P}=-\frac{192 \pi}{5}\left(\frac{2 \pi \mathrm{T}_{\odot}}{P}\right)^{5 / 3}\left(1+\frac{73}{24} e^{2}+\frac{37}{96} e^{4}\right)\left(1-e^{2}\right)^{-7 / 2} \frac{M_{p} M_{c}}{M^{1 / 3}} .
$$


The inclination angle can be constrained by measurements of two or more of these effects. However, only in extremely compact systems is this precisely possible. Otherwise, additional effects, such as an eclipse or limits obtained from the lack of an eclipse, or Shapiro time delay, which is caused by the propagation of the pulsar signal through the gravitational field of its companion, must be observed. The Shapiro ${ }^{[6]}$ delay in general relativity produces a delay in pulse arrival time $\frac{7 / 8}{8}$

$$
\delta_{S}(\phi)=2 M_{c} \mathrm{~T}_{\odot} \ln \left[\frac{1+e \cos \phi}{1-\sin (\omega+\phi) \sin i}\right],
$$

where $\phi$ is the true anomaly, the angular parameter defining the position of the pulsar in its orbit relative to the periastron. $\delta_{S}$ is a periodic function of $\phi$ with an approximate amplitude,

$$
\Delta_{S} \simeq 2 M_{c} T_{\odot}\left|\ln \left[\left(\frac{1+e \sin \omega}{1-e \sin \omega}\right)\left(\frac{1+\sin i}{1-\sin i}\right)\right]\right|
$$

which is large only for edge-on binaries, $\sin i \sim 1$ or those which have both large eccentricities and large magnitudes of $\sin \omega$. Only a fraction of pulsars in binaries have two or more sufficiently well-measured relativistic effects to enable precise measurements of the pulsar mass $M_{p}$.

In some cases, the companion star can be optically detected. In most cases, the companion is a white dwarf. It is possible to estimate white dwarf masses from observations of the optical flux, effective temperature and distance, with the latter being especially problematic. Another possibility is to measure the surface gravity from spectral measurements, but currently this is subject to large systematic effects.

Less accurate measurements of neutron star masses are possible in X-ray binaries, in which X-rays are emitted by matter accreting onto a neutron star from a companion star. Both X-ray and optical observations can yield both mass functions $f_{p}$ and $f_{c}$, but $f_{p}$ is subject to large uncertainties due to the faintness of optical radiation. In the case of the black widow pulsar, PSR B1957+20, optical observations of the companion yield both a mass function and an estimate of the inclination $i$ from the shape of the light curve $\frac{9}{9}$. The inferred semimajor axis of the companion's orbit has to be corrected for the finite size of the companion, however (see below), introducing a source of systematic error in the pulsar mass determination of this system. Nevertheless, interesting lower limits to the pulsar mass are obtained 49 .

\section{Recent Measurements}

The most recent information, as of November 2010, is summarized in Figure 1 and Table 1 and collects information from Refs. 10 to 50 . This compilation represents a significant update to the figure of observed masses and references in Ref. 52 .

There is now ample observational support from pulsars for neutron stars with masses significantly greater than $1.5 \mathrm{M}_{\odot}$. These include PSR J1903+0327 which has a main-sequence companion; the pulsars I and $\mathrm{J}$ in the globular cluster Ter 5 , the 


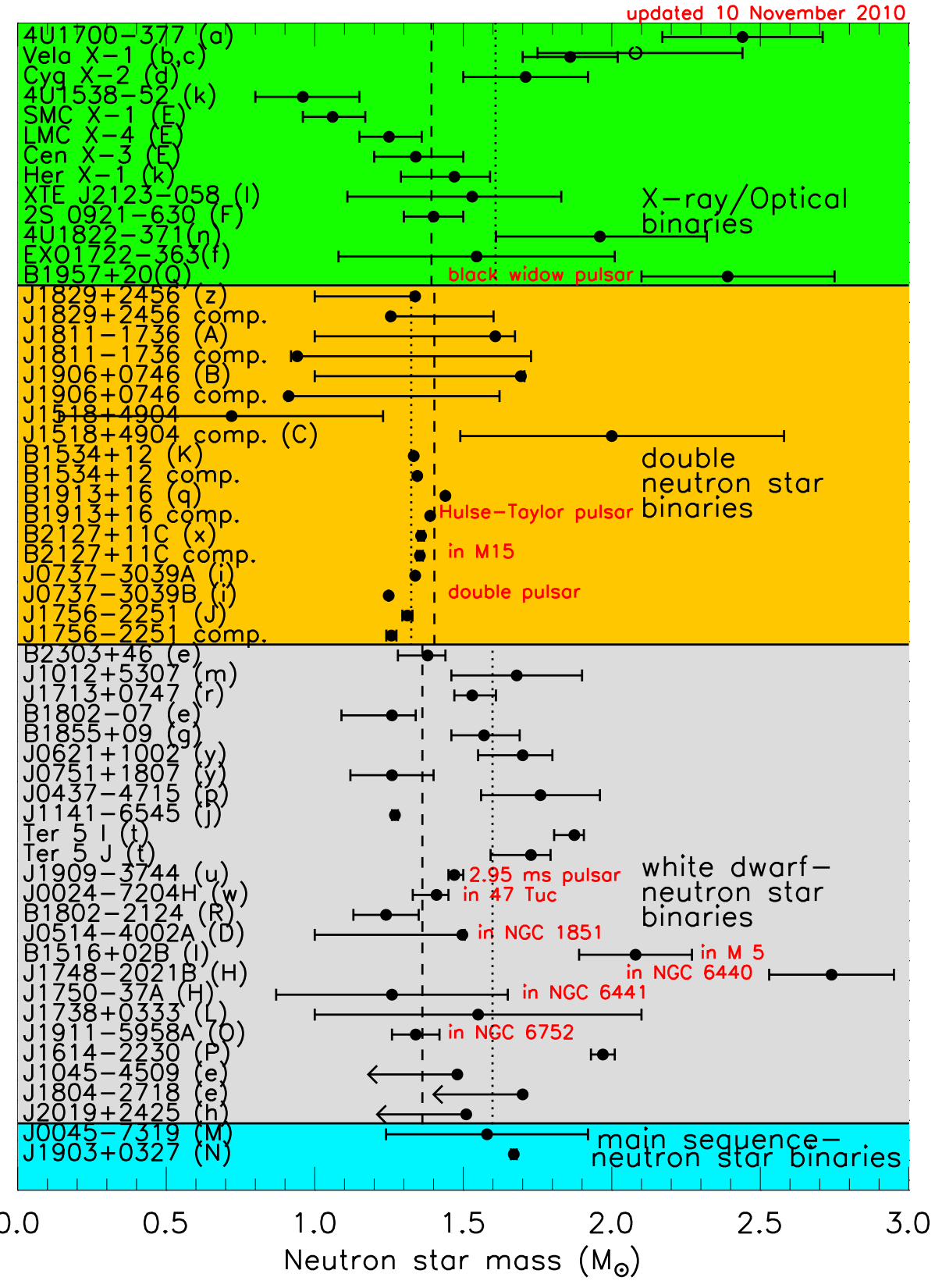

Fig. 1. Measured neutron star masses. References in parenthesis following source numbers are identified in Table 1 
Table 1. Neutron star mass measurements with $1 \sigma$ uncertainties. Reference letters correspond to Fig. 1. For each group, mean and weighted masses are indicated.

\begin{tabular}{|c|c|c|c|c|c|}
\hline Object & Mass $\left(\mathrm{M}_{\odot}\right)$ & Reference & Object & Mass $\left(\mathrm{M}_{\odot}\right)$ & Reference \\
\hline \multicolumn{6}{|c|}{ X-Ray/Optical Binaries $\left(\right.$ mean $=1.609 M_{\odot}$, weighted mean $\left.=1.393 M_{\odot}\right)$} \\
\hline $4 \mathrm{U} 1700-377$ & $2.44_{-0.27}^{+0.27}$ & a $(10)$ & Vela X-1 & $1.86_{-0.16}^{+0.16}$ & $\mathrm{~b}, \mathrm{c}(11,12)$ \\
\hline Cyg X-2 & $1.71_{-0.21}^{+0.21}$ & $\mathrm{~d}(13)$ & $4 \mathrm{U} 1538-52$ & $0.96_{-0.16}^{+0.19}$ & $\mathrm{k}(14)$ \\
\hline SMC X-1 & $1.06_{-0.10}^{+0.11}$ & $\mathrm{E}(\overrightarrow{15})$ & LMC X-4 & $1.25_{-0.10}^{+0.11}$ & $\mathrm{E}(\overrightarrow{15})$ \\
\hline Cen X-3 & $1.34_{-0.14}^{+0.16}$ & $\mathrm{E}(\underline{15})$ & Her X-1 & $1.47_{-0.18}^{+0.12}$ & $\mathrm{k}(14)$ \\
\hline XTE J2123-058 & $1.53_{-0.42}^{+0.30}$ & $1(16,17)$ & 2S 0921-630 & $1.4_{-0.1}^{+0.1}$ & $\mathrm{~F}(18)$ \\
\hline $4 \mathrm{U} 1822-371$ & $1.96_{-0.35}^{+0.36}$ & $\mathrm{n}(19)$ & EXO $1722-363$ & $1.545_{-0.465}^{+0.465}$ & $\mathrm{f}(\overrightarrow{47)}$ \\
\hline B1957+20 & $2.39_{-0.29}^{+0.36}$ & Q (49) & & & \\
\hline \multicolumn{6}{|c|}{ Neutron Star - Neutron Star Binaries $\left(\right.$ mean $=1.325 M_{\odot}$, weighted mean $\left.=1.403 M_{\odot}\right)$} \\
\hline $\mathrm{J} 1829+2456$ & $1.338_{-0.338}^{+0.002}$ & z (20) & $\mathrm{J} 1829+2456(\mathrm{c})$ & $1.256_{-0.003}^{+0.346}$ & $\mathrm{z}(20)$ \\
\hline J1811-1736 & $1.608_{-0.608}^{+0.066}$ & $\mathrm{~A}(\overrightarrow{21})$ & $\mathrm{J} 1811-1736$ (c) & $0.941_{-0.021}^{+0.0787}$ & $\mathrm{~A}(\overrightarrow{21})$ \\
\hline $\mathrm{J} 1906+07$ & $1.694_{-0.694}^{+0.012}$ & B $(\overline{22})$ & $\mathrm{J} 1906+07$ (c) & $0.912_{-0.004}^{+0.710}$ & B $(\overline{22})$ \\
\hline $\mathrm{J} 1518+4904$ & $0.72_{-0.58}^{+0.51}$ & $\mathrm{C}(23)$ & $\mathrm{J} 1518+4904$ (c) & $2.00_{-0.51}^{+0.58}$ & $\mathrm{C}(23)$ \\
\hline $1534+12$ & $1.3332_{-0.0010}^{+0.0010}$ & $\mathrm{~K}(24)$ & $1534+12(\mathrm{c})$ & $1.3452_{-0.0010}^{+0.0010}$ & $\mathrm{~K}(24)$ \\
\hline $1913+16$ & $1.4398_{-0.0002}^{+0.0002}$ & $\mathrm{q}(\overrightarrow{25)}$ & $1913+16(\mathrm{c})$ & $1.3886_{-0.0002}^{+0.0002}$ & $\mathrm{q}(\overrightarrow{25)}$ \\
\hline $2127+11 \mathrm{C}$ & $1.358_{-0.010}^{+0.010}$ & $\mathrm{x}(26)$ & $2127+11 \mathrm{C}(\mathrm{c})$ & $1.354_{-0.010}^{+0.010}$ & $\mathrm{x}(26)$ \\
\hline J0737-3039A & $1.3381_{-0.0007}^{+0.0007}$ & i $(27)$ & J0737-3039B & $1.2489_{-0.0007}^{+0.0007}$ & i $(27)$ \\
\hline $\mathrm{J} 1756-2251$ & $1.312_{-0.017}^{+0.017}$ & $\mathrm{~J}(28)$ & $\mathrm{J} 1756-2251$ (c) & $1.258_{-0.017}^{+0.017}$ & $\mathrm{~J}(28)$ \\
\hline \multicolumn{6}{|c|}{ Neutron Star - White Dwarf Binaries $\left(\right.$ mean $=1.599 M_{\odot}$, weighted mean $\left.=1.362 M_{\odot}\right)$} \\
\hline $\mathrm{B} 2303+46$ & $1.38_{-0.10}^{+0.06}$ & e (29) & $\mathrm{J} 1012+5307$ & $1.68_{-0.22}^{+0.22}$ & $\mathrm{~m}(30)$ \\
\hline $\mathrm{J} 1713+0747$ & $1.53_{-0.04}^{+0.08}$ & r (31, 51) & B1802-07 & $1.26_{-0.17}^{+0.08}$ & e (29) \\
\hline B1855+09 & $1.57_{-0.11}^{+0.12}$ & $\mathrm{~g}(32 \sqrt{51})$ & J0621+1002 & $1.70_{-0.17}^{+0.10}$ & y $(33)$ \\
\hline J0751+1807 & $1.26_{-0.14}^{+0.14}$ & y 33 & J0437-4715 & $1.76_{-0.20}^{+0.20}$ & $\mathrm{p}(34)$ \\
\hline J1141-6545 & $1.27_{-0.01}^{+0.01}$ & $\mathrm{j}(35)$ & Ter $5 \mathrm{I}$ & $1.874_{-0.068}^{+0.32}$ & $\mathrm{t}(\underline{36})$ \\
\hline Ter $5 \mathrm{~J}$ & $1.728_{-0.136}^{+0.066}$ & $\mathrm{t}(\overrightarrow{36})$ & J1909-3744 & $\begin{array}{l}1.47_{-0.02}^{+0.03} \\
x^{\circ}\end{array}$ & $\mathrm{u}(\overrightarrow{37})$ \\
\hline J0024-7204H & $\begin{array}{l}1.41_{-0.08}^{+0.04} \\
x^{2}\end{array}$ & $\mathrm{w}(38)$ & B1802-2124 & $1.24_{-0.11}^{+0.11}$ & $\mathrm{R}(\overline{50})$ \\
\hline J0514-4002A & $1.497_{-0.497}^{+0.008}$ & D (39) & $\mathrm{B} 1516+02 \mathrm{~B}$ & $2.08_{-0.19}^{+0.19}$ & I (40) \\
\hline J1748-2021B & $2.74_{-0.21}^{+0.21}$ & $\mathrm{H}(41)$ & J1750-37A & $1.26_{-0.39}^{+0.39}$ & $\mathrm{H}(41)$ \\
\hline $\mathrm{J} 1738+0333$ & $1.55_{-0.55}^{+0.55}$ & $\mathrm{~L}(42)$ & J1911-5958A & $1.34_{-0.08}^{+0.08}$ & $\mathrm{O}(43)$ \\
\hline J1614-2230 & $1.97_{-0.04}^{+0.04}$ & $\mathrm{P}(48)$ & J1045-4509 & $<1.48$ & e (29) \\
\hline J1804-2718 & $<1.70$ & e $(29)$ & $\mathrm{J} 2019+2425$ & $<1.51$ & $\mathrm{~h}(\underline{44}$ \\
\hline \multicolumn{6}{|c|}{ Neutron Star - Main Sequence Binaries } \\
\hline J0045-7319 & $1.58_{-0.34}^{+0.34}$ & M (45) & J1903+0327 & $1.67_{-0.01}^{+0.01}$ & N (46) \\
\hline
\end{tabular}

globular cluster binaries PSR J1748-2021B and PSR B1516+02B, and PSR J16142230, all with white-dwarf companions; and the black widow pulsar B1957+20. The inclination angles of the two binaries containing pulsars I and J in the globular cluster Ter 5 are unconstrained by observation, but if their inclinations are individually randomly directed, there is a $95 \%$ chance that at least one of these pulsars is greater than $1.68 \mathrm{M}_{\odot} 36$. Assumptions related to random orientation also have roles in the mass determinations for PSR J1748-2021B and PSR B1516+02B. One has to be careful about the assumption of random orientation, however, as the selection of 
potential candidates for follow-up observations necessary for accurate mass determinations are biased. The case of PSR J0751+1807 illustrates the point: initially estimated to have a mass $2.2 \pm 0.2 \mathrm{M}_{\odot}$, the mass was recently established to be $1.26 \mathrm{M}_{\odot}$ upon measurement of another relativistic parameter. Nevertheless, about 2 years ago, a measurement of the inclination of PSR J1903+0327 $\frac{46}{46}$ using Shapiro time delay lead to an accurately determined mass of $1.67 \pm 0.01 \mathrm{M}_{\odot}$, replacing PSR B1913+16 as establishing the value for the minimum maximum mass.

But even more outstanding is the recent determination of $1.97 \pm 0.04 \mathrm{M}_{\odot}$ for the mass of PSR J1614-2230 $\underline{48}$, also using Shapiro time delay to measure the inclination. This $3.15 \mathrm{~ms}$ pulsar is in an $8.7 \mathrm{~d}$ nearly circular orbit about an $0.5 \mathrm{M}_{\odot}$ companion, with $a_{p} \sin i=11.3$ light-second and $\sin i=0.99989$, i.e., it is almost edge on. The Shapiro time delay amplitude, from Eq. ([6), becomes

$$
\Delta_{s} \simeq 4 M_{c} T_{\odot} \ln (2 / \cos i) \simeq 48.8 \mu \mathrm{s} .
$$

By virtue of its accuracy, this mass measurement has become the new standard for the minimum value of the neutron star maximum mass.

In addition, a number of X-ray binaries seem to contain high-mass neutron stars: about $1.9 \mathrm{M}_{\odot}$ in the case of Vela X-1 and $2.4 \mathrm{M}_{\odot}$ in the case of $4 \mathrm{U}$ 1700-377. Nonetheless, the large systematic errors inherent in X-ray binary mass measurements warrant caution.

The black widow pulsar represents an intriguing case, with a best estimate of about $2.4 \mathrm{M}_{\odot}$. This system has both pulsar timing and optical light curve information. It consists of a $1.6 \mathrm{~ms}$ pulsar in a nearly circular $9.17 \mathrm{~h}$ orbit around an extremely low mass companion, $M_{c} \simeq 0.03 \mathrm{M}_{\odot}$. The pulsar is eclipsed for about 50-60 minutes of each orbit, but considering that $a_{p} \sin i=0.089$ light-second $=$ $0.038 \mathrm{R}_{\odot}$, and $a_{c} \sin i \sim 3 \mathrm{R}_{\odot}$ is $M_{p} / M_{c} \simeq 80$ times larger, the eclipsing object has to be much larger $\left(\sim 3 \mathrm{R}_{\odot} / 10\right)$ than the size of the companion star. It is believed that irradiation of the companion by the pulsar strongly heats its nearside to the point of ablation, leading to a comet-like tail and a large cloud of plasma which is responsible for the eclipsing. The pulsar is literally consuming its companion, hence the name "black widow", and has reduced its mass to a small fraction of its original mass. The irradiation also produces an enormous (factor of 100) variation in the brightness of the companion depending on how much of its nearside is visible during its orbit. The companion is bloated and nearly fills its Roche lobe. The companion's optical light curve allows the mass ratio $M_{p} / M_{c}$ and the inclination angle $i$ to be estimated. However, the large size of the companion means that the "center of light" of the system is not equivalent to its "center of mass": the optical light curve depends on the projected semi-major axis of the irradiated nearside of the companion rather than the projected semi-major axis of the center of mass of the companion. The extreme cases are either that the companion has zero radius or that it completely fills its Roche lobe, but estimates based on modeling considerably reduce the allowed range. The extremes lead to a range $1.7<M_{p} / \mathrm{M}_{\odot}<3.2$, but the likely value is $2.4 \pm 0.4 \mathrm{M}_{\odot}$. It will clearly be valuable to extend observa- 
tions and modeling of this system since a $2.4 \mathrm{M}_{\odot}$ neutron star would have profound implications.

\section{What Gerry Would Say}

We would love to be able to argue with Gerry about these observations and their implications, and so we felt this was an ideal topic to explore in this contribution. We can, however, guess what Gerry would have said: "We can't tell nature how to behave; we have to explain it" and, as he often quoted Bismarck, "Was schert mich mein Geschwaetz von gestern?" ("Why do I care about my twaddle from yesterday?")

\section{What We Say}

One of the most surprising consequences of a large pulsar mass measurement is that it severely restricts values of the maximum central density and pressure in that star, and, by extension, that of any other neutron star as well. This realization was elaborated in Ref. [52, but it can be extended to restrict the possibilities for the presence of exotic matter, such as hyperons, Bose condensates and/or quark matter, in neutron star interiors. Moreover, we show here that upper limits to the chemical potentials of quark or baryonic matter can be established using the available mass data, and that these are smaller than often employed in calculations of the properties of quark matter. The consequences of well-determined large neutron star masses is the topic to which we now turn our attention.

\section{Neutron Star Structure}

The global properties of neutron stars are determined, assuming general relativity, by the Tolman-Oppenheimer-Volkov (TOV) equations 53

$$
\begin{aligned}
\frac{d p}{d r} & =-\frac{G(\varepsilon+p)\left(m+4 \pi r^{3} p / c^{2}\right)}{r\left(r c^{2}-2 G m\right)}, \\
\frac{d m}{d r} & =4 \pi r^{2} \varepsilon / c^{2} \\
\frac{d \mathcal{N}}{d r} & =4 \pi r^{2} n\left(1-\frac{2 G m}{r c^{2}}\right)^{-1 / 2},
\end{aligned}
$$

where $m$ is the enclosed gravitational mass, $\mathcal{N}$ is the enclosed baryon number, $p$ is the pressure, $\varepsilon$ is the total energy density, $n$ is the baryon number density, and $r$ is the radial variable. Choosing a value of the pressure or baryon or energy density at the star's center, and integrating to the surface where the pressure vanishes, results in a specific mass and radius, as shown in Fig. 2. A consequence of general relativity is the existence of a maximum mass: configurations with larger central pressures are dynamically unstable and collapse into black holes. 

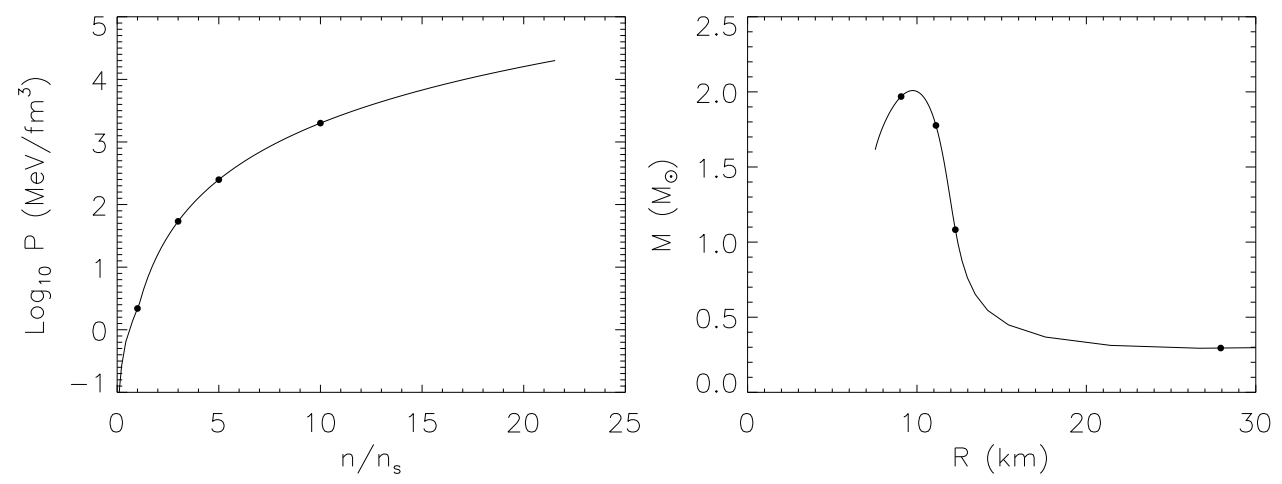

Fig. 2. Solution of the TOV equations. Integrations beginning at the star's center with pressures and baryon number densities (in units of the nuclear saturation density $n_{s}$ ) shown by the dots in the left panel result in specific masses and radii, shown by the corresponding dots in the right panel.

It is convenient to recast the TOV equations in terms of the dimensionless variable $h$ defined by $d h=d p /(\varepsilon+p)=d \mu / \mu$ where $\mu=(\varepsilon+p) / n$ is the baryon chemical potential, which in beta equilibrium is the same as the neutron chemical potential. Therefore,

$$
\mu=\mu_{o} e^{h},
$$

where $\mu_{o}=\varepsilon_{o} / n_{o}, \varepsilon_{o}$ and $n_{o}$ are the chemical potential, energy density and baryon number density, respectively, where $p=h=0$. For a star with a normal crust, $\mu_{o} \simeq 930 \mathrm{MeV}$, the approximate mass-energy of iron nuclei per baryon. Note that the quantity $h$ is related to the metric function $\nu$, where $g_{t t}=e^{\nu}$ and $d h=-d \nu / 2$. One finds

$$
\begin{aligned}
\frac{d r^{2}}{d h} & =-\frac{2 r^{2} c^{2}}{G}\left[\frac{r c^{2}-2 G m}{m c^{2}+4 \pi r^{3} p}\right], \\
\frac{d m}{d h} & =-\frac{4 \pi r^{3} \varepsilon}{G}\left[\frac{r c^{2}-2 G m}{m c^{2}+4 \pi r^{3} p}\right], \\
\frac{d \mathcal{N}}{d h} & =-\frac{4 \pi r^{7 / 2} n c^{3}}{G}\left[\frac{\sqrt{r c^{2}-2 G m}}{m c^{2}+4 \pi r^{3} p}\right] .
\end{aligned}
$$

Writing the TOV equations this way ensures that near both the center and the surface the right-hand sides are finite. The equations are integrated from a central value $h=h_{c}$ to $h=0$. In order to obtain a specific total gravitational mass $M=$ $m(h=0)$, one iterates to find the appropriate value of $h_{c}$. For a given EOS, that is, the functions $\varepsilon(h)$ and $p(h)$, a specific value or $h_{c}, h_{\max }$, results in the maximum mass configuration where $M=M_{\max }, N=N_{\max }$ and $R=R_{\max }$ where $R=r(h=$ $0)$ is the stellar radius and $N=\mathcal{N}(h=0)$ is the total baryon number. The baryon 


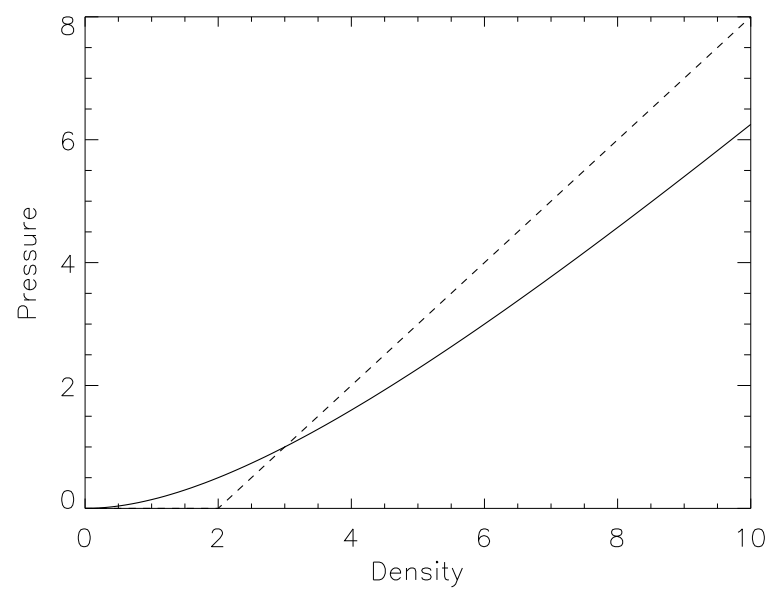

Fig. 3. The solid line is a typical neutron star EOS. The dashed line shows the maximally compact EOS formed by minimizing the pressure $p$ at low energy density $\varepsilon$ (i.e., $p=0)$ and maximizing $p$, subject to causality, at high density (i.e., $\left.p=\varepsilon-\varepsilon_{o}\right) . p$ and $\varepsilon$ are in the same arbitrary units, with $\varepsilon_{o}$ in this example set to 2 .

number is useful in the definition of the binding energy

$$
B E=\left(N m_{b}-M\right) c^{2},
$$

where $m_{b}$ is the baryon mass.

It is an inescapable consequence of general relativity that all EOSs result in a maximum mass configuration, in contrast to Newtonian gravity for which no such limit exists. It is intuitive that the largest maximum masses will be a consequence of the stiffest EOSs, i.e., those with the largest pressures for a given density. It is commonly assumed that the maximum realistic limit to the pressure is obtained by assuming causality, in other words, that the sound speed is limited everywhere by light speed. The assumption of causality led Rhoades and Ruffini 54 to their estimate for the maximum neutron star mass. It is logical, therefore, that the maximum mass configuration so obtained must also have the largest possible values for the central pressure, energy density and chemical potential. The main motivation of this paper is to explore the consequences of this in light of new observations of large neutron star masses.

It was argued by Haensel and Zdunik $[55$ and by Koranda, Stergioulas and Friedman 56 that the most compact configurations result from the combination of a soft EOS at small densities and a stiff EOS at large densities. The extreme limit of this situation is shown in Fig. 3, where the pressure vanishes below the density $\varepsilon_{o}$ and is assumed causal at all higher densities (the so-called maximally compact EOS):

$$
p=0 \quad \varepsilon \leq \varepsilon_{o}, \quad p=\varepsilon-\varepsilon_{o} \quad \varepsilon \geq \varepsilon_{o} .
$$

Since $\varepsilon_{o}$ is the only EOS parameter, the TOV equations scale with it according 
to 57

$$
\varepsilon \propto \varepsilon_{o}, \quad p \propto \varepsilon_{o}, \quad n \propto \varepsilon_{o}, \quad m \propto \varepsilon_{o}^{-1 / 2}, \quad r \propto \varepsilon_{o}^{-1 / 2} .
$$

Following Lattimer, Prakash, Masak and Yahil 58 (hereafter LPMY), we wish to study a somewhat more general EOS, which encompasses the maximally compact EOS as a special case, namely,

$$
p=s\left(\varepsilon-\varepsilon_{o}\right),
$$

where $s$ is a constant representing the square of the adiabatic sound speed. We can then express $\varepsilon, p$ and the baryon number density $n$ in terms of $h$ as

$$
\varepsilon=t^{-1} \varepsilon_{o}\left(s^{-1} e^{h t}+1\right), \quad p=t^{-1} \varepsilon_{o}\left(e^{h t}-1\right), \quad n=\varepsilon_{o} e^{h / s} / \mu_{o}
$$

where $t=1+1 / s$. Using the dimensionless variables

$$
x=r^{2} \epsilon_{o} G / c^{4}, \quad y=m \epsilon_{o}^{1 / 2} G^{3 / 2} / c^{4}, \quad z=\mathcal{N} \epsilon_{o}^{1 / 2} \mu_{o} G^{3 / 2} / c^{6}
$$

the scale-free TOV equations become

$$
\begin{aligned}
& \frac{d x}{d h}=-\frac{2 x\left(x^{1 / 2}-2 y\right)}{y+4 \pi x^{3 / 2} p / \varepsilon_{o}}, \\
& \frac{d y}{d h}=2 \pi x^{1 / 2} \frac{\varepsilon}{\varepsilon_{o}} \frac{d x}{d h}, \\
& \frac{d z}{d h}=2 \pi \frac{n \mu_{o}}{\varepsilon_{o}} \frac{x}{\left(x^{1 / 2}-2 y\right)^{1 / 2}} \frac{d x}{d h} .
\end{aligned}
$$

For a given value of $s$, the value of $h_{\max }$ is the value of $h_{c}$ that maximizes the total gravitational mass $y(h=0)$. By virtue of Eq. (11), $h_{\max }$ then determines the maximum possible chemical potential for that EOS. Table 2 summarizes the properties of the maximum mass solutions for the cases $s=1,4 / 5,2 / 3$ and $s=1 / 3$. The case $s=1$ represents the maximally compact star; as discussed in $\S 4$, the case $s=1 / 3$ is potentially applicable to quark stars or stars with quark cores. For each $s$ are listed the central values of $h, \varepsilon$ and $p$, and the surface values of $x, y$ and $z$, together with other dimensionless quantities of interest.

We will demonstrate that the maximally compact and the $s=1 / 3$ EOSs result in useful approximate limits for the maximum values of thermodynamic quantities in the interior of neutron and quark stars as well as their maximum masses.

\section{Maximally Compact EOS}

We first examine the maximally compact EOS, i.e., the case $s=1$. Expressing the results for $s=1$ shown in Table 2 in astrophysical units (note that $1 \mathrm{MeV}$ 
$\mathrm{fm}^{-3}=1.323 \times 10^{-6} \mathrm{~km}^{-2}$, and $1 \mathrm{M}_{\odot}=1.477 \mathrm{~km}$ ), we find

$$
\begin{aligned}
M_{\max } & =y_{\max } c^{4} \epsilon_{o}^{-1 / 2} G^{-3 / 2}=4.09\left(\varepsilon_{s} / \varepsilon_{o}\right)^{1 / 2} \mathrm{M}_{\odot}, \\
N_{\max } m_{b} & =z_{\max } m_{b} c^{6} \epsilon_{o}^{-1 / 2} \mu_{o}^{-1} G^{-3 / 2}=5.41\left(m_{b} c^{2} / \mu_{o}\right)\left(\varepsilon_{s} / \varepsilon_{o}\right)^{1 / 2} \mathrm{M}_{\odot}, \\
R_{\max } & =x_{\max } c^{2} \epsilon_{o}^{-1 / 2} G^{-1 / 2}=17.07\left(\varepsilon_{s} / \varepsilon_{o}\right)^{1 / 2} \mathrm{~km}, \\
\varepsilon_{\max } & =\left(e^{2 h_{\max }}+1\right) \varepsilon_{o} / 2=3.034 \varepsilon_{o}, \\
p_{\max } & =\left(e^{2 h_{\max }} X S-1\right) \varepsilon_{o}=2.034 \varepsilon_{o}, \\
\mu_{\max } & =\mu_{o} e^{h_{\max }}=2.251 \mu_{o}, \\
n_{\max } & =\left(\varepsilon_{\max }+p_{\max }\right) e^{-h_{\max }} / \mu_{o}=2.251\left(\varepsilon_{o} / \mu_{o}\right) .
\end{aligned}
$$

where $\varepsilon_{s} \simeq m_{b} n_{s} \simeq 150 \mathrm{MeV}$ is the mass-energy density at normal nuclear saturation baryon density $n_{s} \simeq 0.16 \mathrm{fm}^{-3}$. The result for $M_{\max }$ is very close to, but slightly smaller than, that discussed by Rhoades \& Ruffini $\stackrel{54}{ }$, the difference being their substitution of a low-density neutron star crust EOS for densities less than $\varepsilon_{f}$, where $\varepsilon_{f}>\varepsilon_{o}$ and $p_{f}>0$. However, the difference is very small, showing the validity of the maximally compact EOS for an approximate limit.

\begin{tabular}{|c|c|c|c|c|c|c|c|c|}
\hline$s$ & $h_{\max }$ & $\varepsilon_{\max } / \varepsilon_{o}$ & $p_{\max } / \varepsilon_{o}$ & $x_{\max }$ & $y_{\max }$ & $z_{\max }$ & $\varepsilon_{\max } y_{\max }^{2} / \varepsilon_{o}$ & $x_{\max }^{1 / 2} / y_{\max }$ \\
\hline 1 & 0.8116 & 3.034 & 2.034 & 0.05779 & 0.08513 & 0.1127 & 0.02199 & 2.824 \\
$1^{* *}$ & 0.855 & 3.00 & 2.00 & 0.0635 & 0.0854 & 0.109 & 0.0219 & 2.95 \\
$4 / 5$ & 0.727 & 3.29 & 1.83 & 0.0535 & 0.0787 & 0.103 & 0.0204 & 2.94 \\
$2 / 3$ & 0.663 & 3.55 & 1.70 & 0.0500 & 0.0732 & 0.0946 & 0.0190 & 3.05 \\
$1 / 2$ & 0.569 & 4.01 & 1.51 & 0.0443 & 0.0644 & 0.0810 & 0.0166 & 3.27 \\
$1 / 3$ & 0.4520 & 4.826 & 1.275 & 0.03649 & 0.05169 & 0.06212 & 0.01289 & 3.696 \\
$1 / 3^{*}$ & 0.4350 & 6.585 & 1.639 & 0.02696 & 0.04307 & 0.05134 & 0.01221 & 3.813 \\
$1 / 3^{* *}$ & 0.491 & 4.68 & 1.23 & 0.0439 & 0.0522 & 0.0607 & 0.0128 & 4.01 \\
\hline
\end{tabular}

The results for $\varepsilon_{\max } / \varepsilon_{o}$ and $p_{\max } / \varepsilon_{o}$ for the case $s=1$ were previously obtained by Ref. [56. What they did not apparently realize was the significance of the quantity $\varepsilon_{\max } y_{\max }^{2} / \varepsilon_{o}$, which, when expressed in astrophysical units for the maximally compact case $s=1$, is

$$
\varepsilon_{\max } M_{\max }^{2}=1.358 \times 10^{16} \mathrm{~g} \mathrm{~cm}^{-3} \mathrm{M}_{\odot}^{2} .
$$

This equation, of course, states that the central energy density of the maximum mass star is inversely proportional to the square of the maximum mass. Since the maximally compact EOS very closely predicts the largest value for the maximum mass, it seems to follow that this equation should also describe the maximum possible energy density as well. In other words, substituting the mass of the largest observed neutron star mass for $M_{\max }$ in the above expression not only determines the largest energy density in that star, but it might yield an approximate upper 


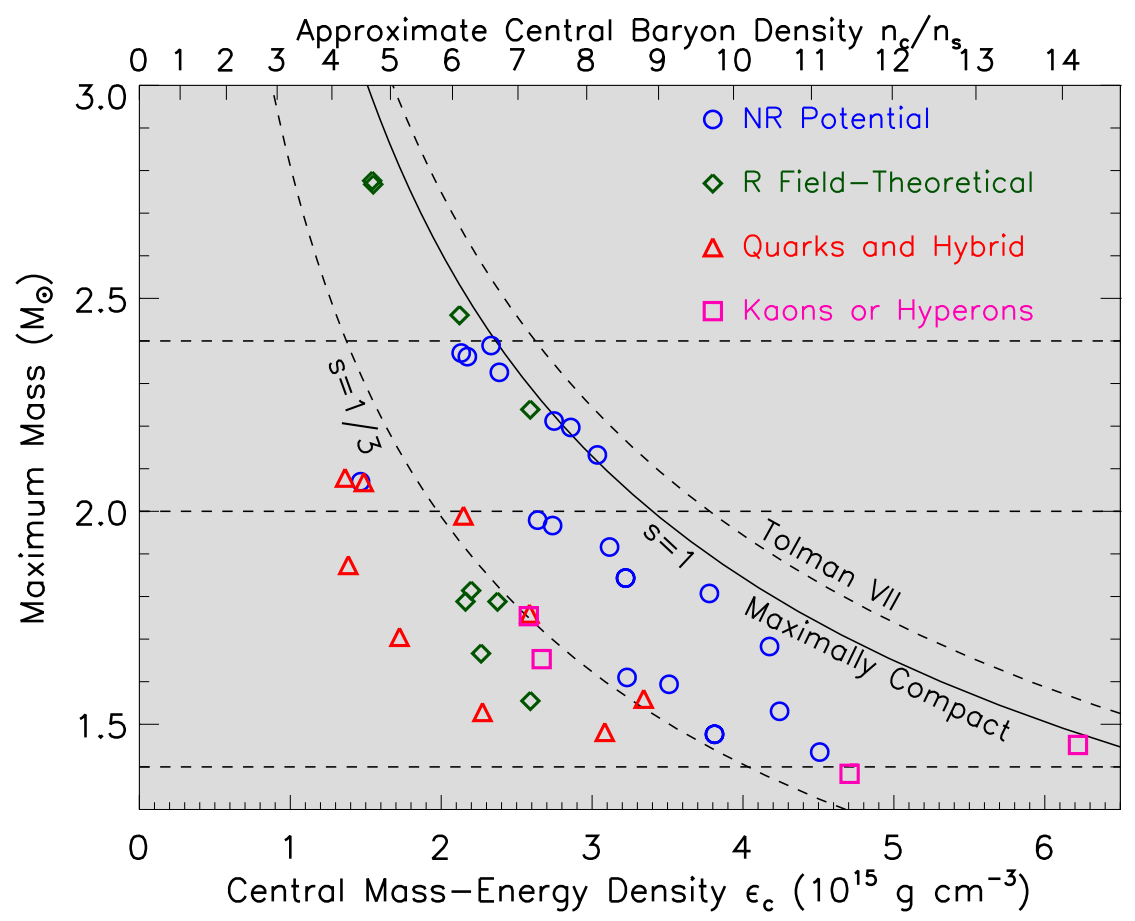

Fig. 4. The central density versus mass relations for the maximally compact $\operatorname{EOS}(s=1$, Eq. (25) in comparison to Tolman VII + causality and the $s=1 / 3$ (Eq. (39)) self-bound cases. Various points are results from different kinds of EOSs, as labelled. Horizontal dashed lines indicate masses of $1.4,2.0$ and $2.4 \mathrm{M}_{\odot}$, respectively.

limit to the energy density to be found in any neutron star. Should a more massive star ever be measured, the upper energy density limit would have to decrease.

It is interesting that the analytic solution to the TOV equations for the energy density distribution

$$
\varepsilon=\varepsilon_{c}\left[1-(r / R)^{2}\right]
$$

i.e., the Tolman VII solution 63, coupled with the causality condition Eq. (27), predicts a value for $\varepsilon_{\max } M_{\max }^{2}$ to within a few percent of Eq. (25), even though the EOS resulting from Eq. (26) becomes acausal at sufficiently large densities. Ref. 52 showed that the limit established from the Tolman VII solution is satisfied phenomenologically by all realistic EOSs, as illustrated in Fig. 4. By comparison, the limit defined by Eq. (25) is slightly violated by two of the EOS models but nevertheless represents a realistic limit. As was the case for the estimate of the maximum neutron star mass, the slight violations result from the assumption of zero pressure at low densities.

Ref. 56 argues that the maximally compact EOS generally predicts the smallest radius for a configuration with arbitrary mass. In this event, the minimum com- 
pactness $R / M$ for any neutron star is determined by

$$
R_{\max } / M_{\max }=2.824 G / c^{2}
$$

a result consistent with discussions by Refs. $59,55,58$ and 60. Modifying the EOS at low densities, i.e., for $\varepsilon<(2-3) \varepsilon_{s}$, does not significantly alter the values for $M_{\max }$, but the values for $R_{\max }$ are moderately increased 58 .

Ref. 56 also demonstrated that the axially symmetric structure equations in general relativity also scale exactly for the maximally compact EOS in terms of $\epsilon_{o}$. The maximum rotation rate, limited by mass-shedding at the equator, scales as $\sqrt{M_{\max } / R_{\max }^{3}} \propto \sqrt{\varepsilon_{o}}$ where the mass and radius refer to the values for maximum mass of the non-rotating configuration. As a result, they calculated the minimum spin period for a causally-limited EOS:

$$
P_{\text {min }}=0.74\left(\frac{\mathrm{M}_{\odot}}{M_{\max }}\right)^{1 / 2}\left(\frac{R_{\max }}{10 \mathrm{~km}}\right)^{3 / 2} \mathrm{~ms}=0.20 \frac{M_{\max }}{\mathrm{M}_{\odot}} \mathrm{ms}
$$

Lattimer and Prakash $\frac{61}{6}$ showed that for realistic equations of state the coefficient $0.74 \mathrm{~ms}$ is effectively replaced by $1.00 \mathrm{~ms}$, a value almost exactly equal to the general relativistic result in the case that the gravitational potential of the rotating star is due to a point mass at the origin 62. Furthermore, the revised formula accurately predicts the maximum rotation rate of any star, not just the maximum mass configuration, if $M_{\max }$ and $R_{\max }$ are replaced by their non-rotating values.

The maximally compact configuration is also expected to maximize the binding energy,

$$
\mathrm{BE}_{\max }=\left(\frac{m_{b} c^{2}}{\mu_{o}} z_{\max }-y_{\max }\right) \frac{c^{6}}{\varepsilon_{o}^{1 / 2} G^{3 / 2}}=0.337 M_{\max } c^{2} .
$$

The maximum binding energy is thus $25.2 \%$ of the rest mass, a fraction that is independent of $\varepsilon_{o}$ and $M_{\max }$.

It is intersting that each of the cases shown in Table 2 yields limiting values for $h_{\max }$ and therefore $\mu_{\max }$ that are also independent of $\varepsilon_{o}$ and therefore also $M_{\max }$. In the case of the maximally compact EOS, one finds the upper limit to the baryon chemical potential

$$
\mu_{\max }=2.251 \times 930 \mathrm{MeV}=2093 \mathrm{MeV} .
$$

These limits to the chemical potential, baryon number density, and binding energy have not been previously calculated. We next explore the implications of the large neutron star masses recently measured, and whether the limits obtained from the ideal $s=1$ and $s=1 / 3$ cases pose realistic upper bounds for thermodynamic quantities within neutron stars.

\section{Implications of the newly-measured mass}

The measured mass of $1.97 \mathrm{M}_{\odot}$ can now be used to set specific limits because the value of $\epsilon_{o}$ is limited according to the first of Eqs. (24): $\varepsilon_{o}<4.33 \varepsilon_{s}$. This implies 
that

$$
\begin{aligned}
& \varepsilon_{\max }<3.034 \times 4.33 \varepsilon_{s}=13.1 \varepsilon_{s}=1.97 \mathrm{GeV} \mathrm{fm}^{-3}, \\
& p_{\text {max }}<2.034 \times 4.33 \varepsilon_{s}=8.81 \varepsilon_{s}=1.32 \mathrm{GeV} \mathrm{fm}^{-3}, \\
& n_{\text {max }}<2.251 \times 4.33 n_{s}=9.75 n_{s}=1.56 \mathrm{fm}^{-3} .
\end{aligned}
$$

Recall that the upper limit to the chemical potential, $\mu_{\max } \simeq 2.23 \varepsilon_{s} / n_{s}=2.1 \mathrm{GeV}$, is independent of the measured mass.

Finally, let us note the implications of a hypothetical measurement of $2.4 \mathrm{M}_{\odot}$, for example, were the current estimate of the black widow pulsar to be confirmed. In this case, by virtue of the first of Eqs. (24), we find $\varepsilon_{o}<2.92 \varepsilon_{s}$. This in turn implies that

$$
\begin{aligned}
& \varepsilon_{\max }<3.034 \times 2.92 \varepsilon_{s}=8.86 \varepsilon_{s}=1.33 \mathrm{GeV} \mathrm{fm}^{-3}, \\
& p_{\text {max }}<2.034 \times 2.92 \varepsilon_{s}=5.94 \varepsilon_{s}=0.89 \mathrm{GeV} \mathrm{fm}^{-3}, \\
& n_{\text {max }}<2.251 \times 2.92 n_{s}=6.57 n_{s}=1.05 \mathrm{fm}^{-3} .
\end{aligned}
$$

\section{Quark Matter EOSs}

As at sufficiently high density a quark-hadron transition is likely to occur, it is reasonable to consider a quark matter EOS for the highest densities that might exist in neutron stars. In the case that quark matter has a lower energy per baryon at zero pressure than iron nuclei, a pure quark star is possible in which case the pressure vanishes at non-zero baryon density 57 . This is an example of a self-bound star. The radii of these stars decrease with decreasing mass in contrast to the case of normal neutron stars. The existence of a thin hadronic crust on such a star is possible, but this would not affect considerations of the maximum mass or central density, pressure or chemical potential of the star, although the mass-radius relation for small masses is dramatically altered.

The term hybrid star refers to the case in which the star's interior contains exotica such as hyperons, Bose condensates and /or quark matter whereas the star's exterior is made up of hadronic matter as in a normal neutron star. The presence of matter extending to vanishing density causes the radii of hybrid stars to increase with decreasing mass as for normal neutron stars. A quark matter EOS is obviously softer than the maximally compact EOS, and since the maximum mass depends primarily on the EOS at the highest densities, the maximal constraints for pure quark stars or for neutron stars with quark cores will be more severe than for the maximally compact case. A relevant question is whether or not the transition to quark matter is at low enough density to occur in a neutron star.

\section{MIT Bag Model}

Quark matter is likely to be highly interacting for densities near the transition density. Nevertheless, the leading order interaction terms are generally attractive 
so that a reasonable upper limit to the pressure of quark matter is that for noninteracting massless quarks,

$$
p=\frac{1}{3}(\epsilon-4 B)
$$

where $B$ is the MIT bag constant (see, e.g., Ref. 64). This is just the EOS of Eq. (18) with $s=1 / 3$ and $\epsilon_{o}=4 B$. Even if QCD corrections are incorporated, the generic form in Eq. (18) is not greatly altered 65. Additional corrections for a non-zero strange quark mass and for pairing introduces attractive contributions $\frac{65}{65}$ and must lower the maximum mass so we ignore these for now. If the EOS of quark matter approaches the ideal of Eq. (33) at high density, and there is significant quark matter in a neutron star interior, we conjecture that limits observed in the case $s=1 / 3$ could more realistically be expected to approximately constrain the maximum mass and maximum possible energy density, pressure and chemical potential than what is determined from the maximally compact EOS. Irrespective of the value for $s$, the relation $h=\ln \left(\mu / \mu_{o}\right)$ is still valid. The limits to mass and compactness were investigated for the case $s=1 / 3$ by LPMY 58 . The results for the dimensionless integrations are contained in Table 2, All results for structural variables scale with $\varepsilon_{o}$ just as in the maximally compact case.

¿From Table 2 it is clear for the case $s=1 / 3$ that the maximum value of $h$, $h_{\max }=0.4520$, is considerably smaller than its value for the maximally compact EOS. The maximum mass stars are also not as compact, the limiting $R / M$ being 3.696. The various dimensionless limits for the case $s=1 / 3$ include

$$
\begin{aligned}
M_{\max } & =2.48\left(\varepsilon_{s} / \varepsilon_{o}\right)^{1 / 2} \mathrm{M}_{\odot}, \\
N_{\max } m_{b} & =2.98\left(m_{b} c^{2} / \mu_{o}\right)\left(\varepsilon_{s} / \varepsilon_{o}\right)^{1 / 2} \mathrm{M}_{\odot}, \\
R_{\max } & =13.56\left(\varepsilon_{s} / \varepsilon_{o}\right)^{1 / 2} \mathrm{~km}, \\
\mu_{\max } & =\mu_{o} e^{h_{\max }}=1.571 \mu_{o}, \\
\varepsilon_{\max } & =\left(e^{2 h_{\max }}+1\right) \varepsilon_{o} / 2=4.826 \varepsilon_{o}, \\
p_{\max } & =\left(e^{2 h_{\max }}-1\right) \varepsilon_{o}=1.275 \varepsilon_{o}, \\
n_{\max } & =\left(\varepsilon_{\max }+p_{\max }\right) / \mu_{\max }=3.884\left(\varepsilon_{o} / \mu_{o}\right) .
\end{aligned}
$$

The results for $M_{\max }, R_{\max }$ and $\varepsilon_{\max }$ were previously obtained by Witten 57. A result of great significance is that, under the reasonable assumption that $\varepsilon_{o}>\varepsilon_{s}$ (otherwise deconfined quark matter would be visible in atomic nuclei), the maximum mass of a pure quark star or a hybrid star with a substantial quark core can be no greater than $2.48 \mathrm{M}_{\odot}$. We will see that, in the case of pure quark stars, more realistic quark models generally have smaller pressures for a given energy density than the MIT bag model and therefore smaller maximum masses. In the case of hybrid stars, if quark matter exists in the cores, the limiting EOS is essentially that of pure quark matter. The magnitude of the transition density $n_{t}$ depends sensitively on whether the hadronic EOS is soft or stiff at the relevant densities. For stiff hadronic EOSs $n_{t}$ is often larger than the central density of the maximum mass star and quarks will 
not appear. In the case that $n_{t}$ is less than several times $n_{s}$, we will show that the hadronic EOS at lower densities does not substantially alter the maximum mass. Thus, the observation of a $2.4 \mathrm{M}_{\odot}$ neutron star, with the certainty that the true maximum mass is larger, will greatly challenge the notion of the existence of quark matter in neutron star interiors.

It is important to note that the chemical potential $\mu=\mu_{o} \ln h$ refers to the baryon chemical potential and $n$ refers to the number density of baryons. For neutral quark matter with three flavors, the quark chemical potential is $1 / 3$ the baryon (neutron) chemical potential and the quark density is 3 times the baryon density. Nevertheless, the value of $n \mu$ is the same for both types of matter. The value of $\mu_{o}$ will depend on assumptions regarding the surface of quark matter stars. It is widely believed that a thin baryonic crust will exist, and in this case, the value of $\mu_{o}$ will be the same as previously considered: Iron at zero pressure with $\mu_{o} \simeq 930 \mathrm{MeV}$. If $\mu_{o}=930 \mathrm{MeV}$, then we immediately find for the case $s=1 / 3$ that $\mu_{\max }=1.46$ $\mathrm{GeV}$. As we show below, this limit for a self-bound MIT bag quark star remains approximately valid for self-bound interacting quark stars and for hybrid quark stars with a hadronic exterior.

Importantly, the TOV equations do not depend on the value for $\mu_{o}$ as can be seen from Eq. (13), but absolute values for the number density and chemical potential do depend on this quantity. In order to discuss separate limits for the chemical potential or number density, one has to have a specific model for the dependence of the energy density on the chemical potential. For massless quarks in the MIT bag model with bag constant $B$, the relation is

$$
\varepsilon=\frac{9}{4 \pi^{2}(\hbar c)^{3}} \mu_{q}^{4}+B
$$

where $\mu_{q}=\mu / 3$ is the quark chemical potential. Each of the three flavors of quarks has the same chemical potential and density in the massless, neutral, case. We can therefore directly use the results from Table 2 using $\varepsilon_{o}=4 B$ to obtain

$$
\begin{aligned}
& \mu_{q o}=\left(\frac{\epsilon_{o} \pi^{2}}{3}\right)^{1 / 4}(\hbar c)^{3 / 4}=268.6\left(\frac{B^{1 / 4}}{141 \mathrm{MeV}}\right) \mathrm{MeV} \\
& n_{q o}=\left(\frac{\varepsilon+p}{\mu}\right)_{o}=\left(\frac{3}{\pi^{2}}\right)^{1 / 4}\left(\frac{\epsilon_{o}}{\hbar c}\right)^{3 / 4}=0.766\left(\frac{B^{1 / 4}}{141 \mathrm{MeV}}\right)^{3} \mathrm{fm}^{-3} .
\end{aligned}
$$

Note that the limiting value for $\mu_{q o}<310 \mathrm{MeV}$ fom the condition of stability of quark matter at zero pressure implies $B^{1 / 4}<163 \mathrm{MeV}$ for the MIT bag model 57 . 


\section{Constraints from the 2-solar-mass star}

We now see that the observed mass of $1.97 \mathrm{M}_{\odot}$ suggests that

$$
\begin{aligned}
\varepsilon_{o} & <(2.48 / 1.97)^{2} \varepsilon_{s}=1.59 \varepsilon_{s}, \quad B^{1 / 4}<146.3 \mathrm{MeV} \\
\mu_{q o} & <\left(\frac{1.59 \varepsilon_{s} \pi^{2}}{3}\right)^{1 / 4}(\hbar c)^{3 / 4}=278.7 \mathrm{MeV} \\
n_{q o} & <\left(\frac{3}{\pi^{2}}\right)^{1 / 4}\left(\frac{1.59 \varepsilon_{s}}{\hbar c}\right)^{3 / 4}=0.856 \mathrm{fm}^{-3}
\end{aligned}
$$

Therefore, the MIT bag model (without strong interaction corrections) cannot explain the $1.97 \mathrm{M}_{\odot}$ star unless strange quark matter is stable. The condition that $\mu_{q o}>310 \mathrm{MeV}$, necessary for iron to be the ground state of matter at zero pressure, implies that the maximum mass is $1.58 \mathrm{M}_{\odot}$ if the MIT bag model is correct.

One also finds

$$
\begin{aligned}
\varepsilon_{\max } & =1.59 \times 4.826 \varepsilon_{s}=7.669 \varepsilon_{s}, \\
p_{\max } & =1.59 \times 1.275 \varepsilon_{s}=2.026 \varepsilon_{s}, \\
\mu_{q, \max } & =1.571 \mu_{q o}=437.8 \mathrm{MeV}, \\
n_{b, \max } & =\frac{p_{\max }+\varepsilon_{\max }}{3 \mu_{q, \max }}=6.926 n_{s},
\end{aligned}
$$

and

$$
\varepsilon_{\max } M_{\max }^{2}=7.91 \times 10^{15} \mathrm{~g} \mathrm{~cm}^{-3} \mathrm{M}_{\odot}^{2} .
$$

This relation is shown in Fig. 4. The $1.97 \mathrm{M}_{\odot}$ star thus limits the energy density to $2.0 \times 10^{15} \mathrm{~g} \mathrm{~cm}^{-3}=1.13 \mathrm{GeV} \mathrm{fm}^{-3}$, just about half the value for the maximally compact EOS. Likewise, the maximum neutron chemical potential is $\mu_{\max }=3 \mu_{q, \max }$ or $1.29 \mathrm{GeV}$ which is considerably less than the absolute upper limit of $2.09 \mathrm{GeV}$ from causality alone.

Note that the reasonable constraint $\varepsilon_{o}>\varepsilon_{s}$ implies $B^{1 / 4}>130.3 \mathrm{MeV}$ in this model. The relatively narrow restrictions on the value of $B$ imply that $248.2 \mathrm{MeV}<$ $\mu_{q o}<278.7 \mathrm{MeV}$. If one further assumes that the baryon density at the surface of the star is at $q$ times the nuclear saturation density, or $n_{q o}=3 q n_{s}$, one has $1.26<q<1.78$. If a neutron star of $2.4 \mathrm{M}_{\odot}$ were to be found, this range would shrink to an extreme degree.

\section{Modifications to the Bag Model}

In the model developed by Alford et al. $\underline{65}$, QCD corrections are included with a parameter $a_{4}$, and the effects of a finite strange quark mass and pairing are included with a term proportional to the parameter $a_{2}$ :

$$
\varepsilon=\frac{9 a_{4}}{4 \pi^{2}(\hbar c)^{3}} \mu_{q}^{4}-\frac{3 a_{2}}{4 \pi^{2}(\hbar c)^{3}} \mu_{q}^{2}+B_{\text {eff }},
$$

where $B_{\text {eff }}$ is an effective bag constant. 
We first consider the case when $a_{2}=0$. Obviously, the introduction of the parameter $a_{4}$ and the replacement of $B$ with $B_{\text {eff }}$ will not affect the structure of the star and the dimensionless results from the case $s=1 / 3$ still apply as for the MIT bag model. The introduction of the parameter $a_{4}$ affects the chemical potential and number densities, however. The quark chemical potential and quark number density at the surface of the star where $p=0$ can now be determined for $a_{2}=0$ (see also Ref. 66):

$$
\begin{aligned}
& \mu_{q o}=\left(\frac{\epsilon_{o} \pi^{2}}{3 a_{4}}\right)^{1 / 4}(\hbar c)^{3 / 4}=268.6\left(\frac{B_{\text {eff }}^{1 / 4}}{141 \mathrm{MeV}}\right) a_{4}^{-1 / 4} \mathrm{MeV} \\
& n_{q o}=\left(\frac{\varepsilon+p}{\mu}\right)_{o}=\left(\frac{3 a_{4}}{\pi^{2}}\right)^{1 / 4}\left(\frac{\epsilon_{o}}{\hbar c}\right)^{3 / 4}=0.766\left(\frac{B_{\text {eff }}^{1 / 4}}{141 \mathrm{MeV}}\right)^{3} a_{4}^{1 / 4} \mathrm{fm}^{-3} .
\end{aligned}
$$

In this case the observed mass of $1.97 \mathrm{M}_{\odot}$ suggests that

$$
\begin{aligned}
& \mu_{q o}<\left(\frac{1.59 \varepsilon_{s} \pi^{2}}{3 a_{4}}\right)^{1 / 4}(\hbar c)^{3 / 4}=278.7 a_{4}^{-1 / 4} \mathrm{MeV} \\
& n_{q o}<\left(\frac{3 a_{4}}{\pi^{2}}\right)^{1 / 4}\left(\frac{1.59 \varepsilon_{s}}{\hbar c}\right)^{3 / 4}=0.856 a_{4}^{1 / 4} \mathrm{fm}^{-3} .
\end{aligned}
$$

It is now evident that $a_{4}<0.656$ is required if both $\mu_{q o}=310 \mathrm{MeV}$ and $M_{\max }>$ $1.97 \mathrm{M}_{\odot}$ in this model. Further, one has

$$
\begin{aligned}
& \mu_{q, \max }=1.571 \mu_{q o}=437.8 a_{4}^{-1 / 4} \mathrm{MeV}, \\
& n_{b, \max }=\frac{p_{\max }+\varepsilon_{\max }}{3 \mu_{q, \max }}=6.926 a_{4}^{1 / 4} n_{s} .
\end{aligned}
$$

The introduction of the $a_{4}$ term does not alter the limiting energy density inferred from the observed $1.97 \mathrm{M}_{\odot}$ star. However, the maximum baryon chemical potential $\mu_{\max }=1.29 / a_{4}^{1 / 4} \mathrm{GeV}$ increases moderately from the noninteracting quark case. Nevertheless, the condition that $\mu_{q o}=310 \mathrm{MeV}$ does not change the limiting value of $\mu_{\max }=1.46 \mathrm{GeV}$ from that case. We note that one expects from theories of interacting quark matter that $a_{4} \sim 0.765$.

The constraint $\varepsilon_{o}>\varepsilon_{s}$ implies $B_{\text {eff }}^{1 / 4}>130.3 \mathrm{MeV}$ in this model, unchanged by the $a_{4}$ parameter. The restriction on the value of $B_{\text {eff }}$ from the observed $1.97 \mathrm{M}_{\odot}$ star now implies that $248.2<\mu_{q o} a^{1 / 4}<278.7 \mathrm{MeV}$. Finally, assuming the baryon density at the surface of the star to be $q n_{s}$, one finds $1.26<q a_{4}^{-1 / 4}<1.78$.

We next have to determine how modifying the quark EOS by introducing the $a_{2}$ term changes these results. The $a_{2}$ term is generally a small correction to the quark EOS. In solving the TOV equation, we may once again scale energy and pressure with $\varepsilon_{o}=4 B_{e f f}$, but there is an additional parameter $a_{2}$. Furthermore, $\varepsilon_{o}$ is no longer the value of $\varepsilon$ when $p=0$. So the introduction of $a_{2}$ is a moderate complication. 
Although there are now 3 parameters affecting the quark EOS, there is a degeneracy in the parameters as far as structural considerations are concerned. We write Eq. (40) in the dimensionless form

$$
\begin{aligned}
& \frac{\epsilon}{B_{\text {eff }}}=\frac{9 a_{4}}{4 \pi^{2}}\left(\frac{\mu_{q}}{B_{\text {eff }}^{1 / 4}}\right)^{4}-\frac{3}{4 \pi^{2}}\left(\frac{a_{2}^{1 / 2}}{B_{\text {eff }}^{1 / 4}}\right)^{2}\left(\frac{\mu_{q}}{B_{\text {eff }}^{1 / 4}}\right)^{2}+1, \\
& \frac{p}{B_{\text {eff }}}=\frac{3 a_{4}}{4 \pi^{2}}\left(\frac{\mu_{q}}{B_{\text {eff }}^{1 / 4}}\right)^{4}-\frac{3}{4 \pi^{2}}\left(\frac{a_{2}^{1 / 2}}{B_{\text {eff }}^{1 / 4}}\right)^{2}\left(\frac{\mu_{q}}{B_{\text {eff }}^{1 / 4}}\right)^{2}-1 .
\end{aligned}
$$

Therefore it is only necessary to consider as parameters the quantities $a_{4}$ and $a_{2}^{1 / 2} / B_{\text {eff }}^{1 / 4}$ in order to establish scaled results for the maximum mass and central pressure and energy density. The value of $\mu_{q o} / B_{\text {eff }}^{1 / 4}$ is a quadratic solution of the equation for $p=0$ from Eq. (44). To compute numerical values for the mass, energy density, pressure and chemical potential, it is then additionally necessary to fix $B_{\text {eff }}$. However, this is not necessary to establish the quantity $\varepsilon_{\max } y_{\max }^{2} / \varepsilon_{o}$ which is independent of $\varepsilon_{o}=4 B_{\text {eff }}$.

We find that varying $a_{4}$ for fixed nonzero $a_{2}^{1 / 2} / B_{\text {eff }}^{1 / 4}$ has small effects on eigenvalues $h_{\max }, x_{\max }, y_{\max }$ and $\varepsilon_{\max } y_{\max }^{2} / \varepsilon_{o}$. Likewise, there are similar small variations of these eigenvalues with varying $a_{2}^{1 / 2} / B_{\text {eff }}^{1 / 4}$ for fixed values of $a_{4}$. In any event, the values of $h_{\max }, x_{\max }$ and $\varepsilon_{\max } y_{\max }^{2} / \varepsilon_{o}$ are largest for the case $a_{4}=1$ and $a_{2}=0$, as seen in Table 2 Furthermore, the increase in the upper limit to the chemical potential resulting from $a_{4}<1$ is approximately canceled by the $a_{2}$ contribution. Therefore, the upper limits to the central density and pressure established by the masssless quark bag model hold without regard to QCD corrections, pairing, or finite strange quark mass. This result is confirmed phenomenologically, as illustrated in Fig. 4 which indicates that the limiting density curve for $s=1 / 3$, to within a few percent, bounds pure quark stars and hybrid stars containing both quark and hadronic matter. As we proceed to show, the addition of a hadronic mantle and/or crust may permit densities slightly larger than that predicted by Eq. (39), just as they increased the densities for some hadronic neutron stars in some cases beyond the $s=1$ curve in Fig. 4

\section{Simple Hybrid Stars}

The thermodynamic limits we have so far considered for quark matter apply to selfbound configurations with non-zero baryon density at their surface or those with a very thin hadronic crust. Hybrid stars consist of a quark core or a quark-hadron mixed phase together with a massive hadronic exterior. The presence of a mixed phase is possible only if the surface tension of quark matter is sufficiently large. We consider here the case where there is no mixed phase and the transition between quark and hadronic matter is a first-order transition at the point where $\mu$ and $p$ in the two phases are equal, with values $\mu_{t}$ and $p_{t}$, respectively. 
We will employ a straightforward example. A reasonable approximation for hadronic pressures in the vicinity of nuclear matter densities for neutron star matter is $p=K \varepsilon^{2}$, where $K \simeq 1.1 \cdot 10^{-4} \mathrm{fm}^{3} \mathrm{MeV}^{-1}$. For $h>h_{t}$ we will use the general form for self-bound matter, Eq. (18). Requiring $\mu$ and $p$ to match at $h_{t}$, we find

$$
\begin{array}{llrl}
\varepsilon=\left(e^{h / 2}-1\right) / K & h<h_{t} \\
\varepsilon=\varepsilon_{0} / t+e^{\left(h-h_{t}\right) t}\left[\varepsilon_{o} / t+\left(e^{h_{t} / 2}-1\right)^{2} / K\right] / s & h>h_{t} .
\end{array}
$$

In general, $\varepsilon$ and $n$ will be discontinuous at $h_{t}$, but we can require them to be continuous if we set

$$
\varepsilon_{o}=\frac{e^{h_{t} / 2}-1}{K}\left[1-\frac{e^{h_{t} / 2}-1}{s}\right] .
$$

This assumption does not have a significant effect on our results, and has the advantage of reducing the number of free parameters in this model to a single one: $h_{t}$, or, equivalently, $p_{t}$. In the case that $h_{t}$ or $p_{t}$ tends to zero, we recover the selfbound cases. $h_{\max }$ increases slowly with $p_{t}$, approximately as $\ln \left(1+p_{t} / p_{s}\right)$. For the case $p_{t}=10 p_{s}$, integration of the TOV equation with this EOS gives values for the quantities in Table 2 in the rows denoted ${ }^{* *}$.

It is observed in both $s=1$ and $s=1 / 3$ cases with added shells and $p_{t}=10 p_{s}$ that $h_{\max }$ modestly increases by about 0.04 , i.e., $\mu_{\max }$ is increased by about $4 \%$. Further increasing $p_{t}$ results in marginally larger values of $h_{\max }$. Because the selfbound cores in both the $s=1$ and $s=1 / 3$ cases are stiffer than is realistic for hadronic and quark matter, respectively, which tends to unrealistically enhance the values of $h_{\max }$, we conclude that the effects of adding a hadronic shell does not result in a significant increase in the central chemical potential, and the values established for the self-bound cases still hold approximately. However, values of $\varepsilon_{\max } y_{\max }^{2} / \varepsilon_{o}$ decrease with the addition of a hadronic shell, so our model is not sufficiently general

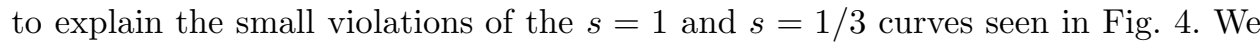
now compare these results, in the case for quark stars, with those from published models.

\section{Published Models of Hybrid Stars}

¿From many calculations in published literature it can be concluded that the density at which exotica appear is uncertain and depends sensitively on the nature of nucleonic interactions up to $(2-3) n_{s}$. In most cases, however, the EOS with exotica is softer than that without. Consequently, the maximum mass that a hybrid star can support is often smaller (with few exceptions) than that of a pure nucleonic star. In view of the observation of a $1.97 \mathrm{M}_{\odot}$ star, the natural question to ask is whether or not exotica are even permitted within such stars.

At the current stage, it is difficult to rule out exotica as many model calculations indicate that a $2 \mathrm{M}_{\odot}$ can indeed be supported by hybrid stars of different varieties. Indeed, Fig. 4 contains examples of hybrid quark stars exceeding this threshhold. 
As another example, Fig. 5 of Ref. 3 shows kaon-condensed stars of $2 \mathrm{M}_{\odot}$ that would be compatible with the current observation. Ref. 67, which contrasts the MIT bag model with the Nambu Jona-Lasinio model for the treatment of quarks, presents results of hybrid stars containing nucleons and quarks, and nucleons, hyperons and quarks (see Figs. 4 of Ref. 67) with maximum masses of $\sim 2 \mathrm{M}_{\odot}$. The central densities of maximum mass configurations obtained there were in the range $(0.7-0.75) \mathrm{fm}^{-3}$ (see Fig. 5 of Ref. 67), which is compatible with the limiting curve $s=1 / 3$ in Fig. 4. It would be interesting to see whether or not Dyson-Schwinger -equation approaches 68 to the quark matter EOS yield similar results.

Recently, perturbative calculations of quark matter (without effects of pairing) including strong interaction effects to order $\alpha_{s}^{2}$ and the effects of the strange quark mass have been performed by Kurkela et al. in Ref. 69. As these authors point out, their results become reliable at the $10 \%$ level only for quark chemical potentials exceeding $1 \mathrm{GeV}$, a value far larger than can exist in pure quark or quark hybrid stars as we have shown (the maximum is about $0.5 \mathrm{GeV}$ ). Their calculations nevertheless result in quark hybrid stars with masses approaching $2 \mathrm{M}_{\odot}$.

These examples show that it is at present not possible to rule out any form of exotica with the $1.97 \mathrm{M}_{\odot}$ observation. It must be stressed, however, that many of these approaches suffer from a great deal of model dependence (even in cases in which a substantially lower than a $2 \mathrm{M}_{\odot}$ maximum mass star is predicted). However, the situation would change dramatically in the event of a confirmed observation of a $2.4 \mathrm{M}_{\odot}$ star. Such an observation would seem to be incompatible with quark or quark hybrid stars including interactions, and would severely restrict models containing other forms of exotica.

\section{Gerry's Views on Hybrid Stars}

Prior to his fascination with kaon condensates, Gerry, together with Hans Bethe and Jerry Coopertsein 70 , had maintained that perturbative QCD cannot be trusted at densities near $n_{s}$ where matter must be confined, and that any strangeness would appear in the form of Lambdas. To infer whether strangeness-rich quark matter can occur at high densities, they calculated the energy density of massless up, down and strange quarks to order $\alpha_{s}$ taking its density dependence as $\alpha_{s}=2.2\left(n / n_{s}\right)^{1 / 3}$. However, as shown in Ref. 66, their choice turned out to be independent of $\alpha_{s}$ once thermodynamic consistency was imposed. The result of their calculations was that the transition to quark matter occurred at densities larger than those found in neutron stars.

A lesson lurks in Gerry's point of view. Most models of hyperon-filled stars have used hyperon-nucleon couplings based on simplistic quark counting in the nonperturbative regime. Analyses of laboratory experiments, however, indicate that at nuclear densities the $\Lambda$-nucleon potential is attractive, but the $\Sigma^{-}$-nucleon potential is repulsive $71 / 72$. Knorren, Prakash and Ellis ${ }^{73}$ investigated the consequences of varying hyperon-nucleon couplings on the structural properties of neutron stars and 
concluded that, if only Lambdas were to exist in neutron stars, their effects on the maximum mass would be minimal. If such is indeed the case, then two birds can be killed with one stone: first, a star with a 2-solar mass could be easily attained, and second, an avenue for a fast cooling could arise even if the direct Urca process with nucleons could not take place as even miniscule amounts of Lambdas through their beta decays can cool the stars rapidly 74 . In a final example, based on a field-theoretical model for the hadrons and an NJL model for the quarks, Ref. 75 fits observed $\Lambda$ and $\Xi^{-}$hyper-nuclei and is able to construct hybrid stars of $2 \mathrm{M}_{\odot}$. Further investigations to fortify these ideas appear promising.

\section{Neutron Matter and Astrophysical Constraints}

It is also possible to place constraints on the maximum mass by combining the condition of causality with laboratory data concerning the symmetry energy, neutron skins on neutron-rich nuclei, and the properties of pure neutron matter as well as with astrophysical measurements of neutron star radii and masses. Recently, estimates of neutron matter properties have been obtained by first principle Green's Function Monte Carlo calculations 76 and from chiral effective interactions 77 . These approaches yield similar results. Hebeler et al. 78 showed that such calculations restrict neutron star radii to be in the range of (11-12) $\mathrm{km}$ when the star's central density is at a density of $1.1 n_{s}$, roughly the uppermost density for which the neutron matter calculations are reliable. With this constraint, it was possible to show that causality limits the maximum mass to about $2.9 \mathrm{M}_{\odot}$ (see Fig. 3 of Ref. 78).

Steiner, Lattimer and Brown 79 demonstrated that estimates of masses and radii of neutron stars in photospheric radius expansion X-ray bursters and also quiescent, cooling neutron stars limit the radii of $1.5 \mathrm{M}_{\odot}$ stars to lie in the radius range (10.9$12.5) \mathrm{km}$. This radius range resulted from a Bayesian analysis of the observational data from three sources of both types. Encouragingly, this radius range is also consistent with the results of neutron matter simulations 76178 . With this massradius constraint, causality limits the maximum mass to about $2.4 \mathrm{M}_{\odot}$, about 0.5 $\mathrm{M}_{\odot}$ smaller than that inferred from the neutron matter results themselves. This upper limit too the maximum mass is due to the fact that the radius does not rapidly change with mass for a significant part of the mass-radius relation, as can be observed in Fig. 2. Thus, a restriction of the radius at a specific mass translates into an upper mass limit when the causality condition (i.e., $M<R c^{2} /(2.9 G)$ ) is imposed.

\section{Conclusions}

Based on the observed largest well-measured mass, we have determined modelindependent upper limits to the central energy density, central pressure and central chemical potential of neutron stars whether or not they are composed of hadrons, 
quarks, or both. If matter undergoes a quark-hadron transition within the density range permitted for neutron star interiors, these limits on central density and chemical potential are substantially lowered. Indeed, if it can be shown that the maximum mass of neutron stars exceeds $2.5 \mathrm{M}_{\odot}$, the possibility of a quark-hadron transition in neutron stars is actually excluded, and the transition in cold matter would have to occur at an energy density in excess of $8 \varepsilon_{s}$ or about $1.2 \mathrm{GeV}$.

We have also demonstrated the existence of an upper limit to the baryon chemical potential, independent of mass observations, of $2.1 \mathrm{GeV}$. If quark matter exists in the cores of neutron stars, the upper limit is likely not greater than $1.5 \mathrm{GeV}$. We also find the maximum binding energy or any neutron star is about $25 \%$ of the rest mass.

The astronomical evidence currently favors the neutron star maximum mass to be between $2 \mathrm{M}_{\odot}$ and $2.5 \mathrm{M}_{\odot}$. A Bayesian analysis of the observed neutron star mass distribution suggests that this distribution shows no indication of being influenced by an absolute upper limit imposed by general relativity and causality 80. In other words, this distribution shows no evidence of the existence of an abrupt upper mass cutoff; rather, the largest observed mass may simply be limited by the amount of mass it is reasonably possible for a neutron star to accrete in a binary. Supernova models generally imply that newly formed neutron stars are not more massive than about $1.5 \mathrm{M}_{\odot}$. In this case it is likely that the true maximum mass determined by the EOS is at least a few tenths of a solar mass larger than the largest observed mass. If so, the chemical potential, density and pressure limits we derived are somewhat conservative. In addition, our survey of EOSs with exotic matter indicates that stars containing significant proportions of this matter in the form of hyperons, Bose condensates or quark matter would be almost impossible.

\section{Acknowlegements}

This research was supported by the Department of Energy under the grants DEAC02-87ER40317 (for JML) and DE-FG02-93ER40756 (for MP). We also value our many discussions with Gerry on topics related to the neutron star maximum mass.

\section{References}

1. D. Kaplan and A. Nelson, Phys. Lett. B175, 57 (1986); B179, 409 (1986).

2. G. E. Brown and H. A. Bethe, Astrophys. J. 423, 659 (1994).

3. V. Thorsson, M. Prakash and J. M. Lattimer, Nucl. Phys. A 572, 693 (1994).

4. A. Burrows, Astrophys. J. 334, 891 (1988).

5. R. N. Manchester and J. H. Taylor, Pulsars (W. H. Freeman, San Francisco, 1977).

6. I. I. Shapiro, Phys. Rev. Lett. 26, 789 (1964).

7. T. Damour and N. Deruelle, Ann. Inst. Henri Poincaré Phys. Theor. 44, 263 (1986).

8. P. C. C. Freire and N. Wex, MNRAS 409, 199 (2010).

9. M. T. Reynolds, P. J. Callanan, A. S. Fruchter, M. A. P. Torres, M. E. Beer and R. A. Gibbons, MNRAS 379, 1117 (2007).

10. J.S. Clark, S. P. Goodwin, P. A. Crowther, L. Kaper, M. Fairbairn, N. Langer, and C. Brocksopp, Astron. \& Astrophys. 392, 909 (2002). 
11. O. Barziv, L. Karper, M.H. van Kerkwijk, J.H. Telging, and J. van Paradijs, Astron. Es Astrophys. 377, 925 (2001).

12. H. Quaintrell, A.J. Norton, T.D.C. Ash, P. Roche, B. Willems, T.R. Bedding, I.K. Baldry and R.P. Fender, Astron. E Astrophys. 401, 303 (2003).

13. J. Casares, J.I. González Hernández, G. Israelian and R. Rebolo, MNRAS 401, 2517 (2010).

14. M.H. van Kerkwijk, J. van Paradijs, and E.J. Zuiderwijk, Astron. E Astrophys. 303, 497 (1995).

15. A.van der Meer, L. Kaper, M. H. van Kerkwijk, M. H. M. Heermskerk and E. P. J. van den Huevel, Astron. \& Astrophys. 473, 523 (2007).

16. D.M. Gelino, J.A. Tomsick and W.A. Heindl, Bull. Am. Astron. Soc. 34, 1199 (2003).

17. J. Tomsick, private communication (2004).

18. D. Steeghs and P. G. Jonker, ApJLetters 669, 85 (2007).

19. P. G. Jonker, M. van der Klis, P. J. Groot, MNRAS 339, 663 (2003).

20. D.J. Champion, D. R. Lorimer, M.A. McLaughlin, K.M. Xilouris, Z. Arzoumanian, P.C.C. Freire, A. N. Lommen, J.M. Cordes and F. Camilo, MNRAS 363, 929 (2005).

21. A. Corongiu, M. Kramer, A.G. Lyne, O. Löhmer, N. D’Amico, \& A. Possenti, Mem. S. A. It Suppl. 5, 188 (2004).

22. D. R. Lorimer et al., ApJ 640, 428 (2006).

23. G.H. Janssen, B.W. Stappers, M. Kramer, D.J. Nice, A. Jessner, I. Cognard and M.B. Purver, Astron. \& Astrophys. 490, 753 (2008).

24. I.H. Stairs, S.E. Thorsett, J.H. Taylor and A. Wolszczan, ApJ 581501 (2002).

25. J. M. Weisberg, D. J. Nice \& J. H. Taylor, ApJ 722, 1030 (2010).

26. B.A. Jacoby, P.B. Cameron, F.A. Jenet, S.B. Anderson, R.N. Murty and S.R. Kulkarni, ApJLetters 644, L113 (2006).

27. M. Kramer, I.H. Stairs, R.N. Manchester, M.A. McLaughlin, A.G. Lyne, R.D. Ferdman, M. Burgay, D.R. Lorimer, A. Possenti, N. D'Amico, J.M. Sarkisian, G.B. Hobbs, J.E. Reynolds, P.C.C. Freire and F. Camilo, Science 314, 97 (2006).

28. R.D. Ferdman, Ph.D. thesis, Univ. of British Columbia (2008).

29. S.E. Thorsett and D. Chakrabarty, Astrophys. J. 512, 288 (1999).

30. Ch. Lange, F. Camilo, N. Wex, M. Kramer, D.C. Backer, A.G. Lyne and O. Doroshenko, MNRAS 326, 274 (2001).

31. E.M. Splaver, D.J. Nice, I.H. Stairs, A.N. Lommen and D.C. Backer, ApJ 620, 405 (2005).

32. D.J. Nice, E.M. Splaver and I.H. Stairs, in Radio Pulsars, ed. M. Bailes, D. J. Nice, and S. E. Thorsett (Ast. Soc. Pac. 302, San Francisco, 2003).

33. D.J. Nice, I.H. Stairs and L.E. Kasian, in 40 YEARS OF PULSARS: Millisecond Pulsars, Magnetars and More, AIP Conference Proceedings 983, 453 (2008).

34. J.P.W. Verbiest, M. Bailes, W. van Straten, G.B. Hobbs, R.T. Edwards, R.N. Manchester, N.D.R. Bhat, J.M. Sarkissian, B.A. Jacoby and S.R. Kulkarni, ApJ 679, 675 (2008).

35. N.D.R. Bhat, M. Bailes and J.P.W. Verbiest, Phys. Rev. D 77, 124017 (2008).

36. S. M. Ransom, J.W.T. Hessels, I.H. Stairs, P.C. Freire, F. Camilo, V.M. Kaspi and K.L. Kaplan, Science 307, 892 (2005).

37. A.W. Hotan, M. Bailes and S.M. Ord, MNRAS 369, 1502 (2006).

38. P. C. C. Freire, F. Camilo, M. Kramer, D.R. Lorimer, A.G. Lyne, R.N. Manchester \& N.D'Amico, MNRAS 340, 1359 (2003).

39. P.C.C. Freire, S.M. Ransom and Y. Gupta, ApJ 662, 1177 (2007).

40. P.C.C. Freire, A. Wolszcan, M. van den Berg and J.W.T. Hessels, ApJ 679, 1433 (2008). 
41. P.C.C. Freire, S.M. Ransom, S. Bégin, I.H. Stairs, J.W.T. Hessels, L.H. Frey and F. Camilo, ApJ 675, 670 (2008).

42. P.C.C. Freire, B.A. Jacoby and M. Bailes, in 40 YEARS OF PULSARS: Millisecond Pulsars, Magnetars and More, AIP Conference Proceedings 983, 488; arXiv:0711.1880 (2008).

43. C.G. Bassa, M. H. van Kerkwijk, D. Koester and F. Verbunt, Astron. \& Astrophys. 456, 295 (2006).

44. D. J. Nice, E. M. Splaver and I. H. Stairs, ApJ 549, 516 (2001).

45. D.J. Nice, IAU Proceedings, Sydney, Australia (2003).

46. P. C. C. Freire, C. G. Bassa, N. Wex, I. H. Stairs, D. J. Champion, S. M. Ransom, P. Lazarus, V. M. Kaspi, J. W. T. Hessels, M. Kramer, J. M. Cordes, J. P. W. Verbiest, P. Podsiadlowski, D. J. Nice, J. S. Deneva, D. R. Lorimer, B. W. Stappers, M. A. McLaughlin and F. Camilo, MNRAS, in press; arXiv:1011.5809 (2010).

47. A. B. Mason, A. J. Norton, J. S. Clark, I. Negueruela \& P. Roche, Astron. \& Astrophys. 509, 79 (2010).

48. P. B. Demorest, T. Pennucci, S. M. Ransom, M. S. E. Roberts and J. W. T. Hessels, Nature, 467, 1081 (2010).

49. M. H. van Kerkwijk, R. Breton \& S. R. Kulkarni, submitted to ApJ (2010); arXiv:1009.5427.

50. R.D. Ferdman, I.H. Stairs, M. Kramer, M.A. McLaughlin, D.R. Lorimer, D.J. Nice, R.N. Manchester, G. Hobbs, A.G. Lyne, F. Camilo, A. Possenti, P.B. Demorest, I. Cognard, G. Desvignes, G. Theureau, A. Faulkner, D.C. Backer, ApJ 711, 764 (2010).

51. T. M. Tauris and G.J. Savonije, Astron. E Astrophys. 350, 928 (1999).

52. J. M. Lattimer and M. Prakash, Phys. Rev. Lett. 94, 111101 (2005).

53. R. C. Tolman, Proc. Nat. Acad. Sci. U. S. A., 20, 3 (1934); J. R. Oppenheimer and G. M. Volkoff, Phys. Rev. 55, 374 (1939).

54. C. E. Rhoades Jr. and R. Ruffini, Phys. Rev. Lett. 32, 324 (1974).

55. P. Haensel and J. L. Zdunik, Nature 340, 617 (1989).

56. S. Koranda, N. Stergioulas and J. L. Friedman, ApJ 488, 799 (1997).

57. E Witten, Phys. Rev. D 30, 272 (1984).

58. J. M. Lattimer, M. Prakash. D. Masak and A. Yahil, ApJ 355, 241 (1990).

59. L. Lindblom, ApJ 278, 364 (1984).

60. N. K. Glendenning, Phys. Rev. D 46, 4161 (1992).

61. J. M. Lattimer and M. Prakash, Science 304, 536 (2004).

62. S. L. Shapiro, S. A. Teukolsky and I. Wasserman, ApJ 272, 702 (1983).

63. R. C. Tolman, Phys. Rev. 55, 364 (1939).

64. E. Fahri and R. L. Jaffe, Phys. Rev. D30, 2379 (1984).

65. M. G. Alford, M. Braby, M. Paris and S. Reddy, ApJ 629, 969 (2005).

66. Manju Prakash, E. Baron and M. Prakash, Phys. Lett. B 243, 175 (1990).

67. A. W. Steiner, M. Prakash and J. M. Lattimer, Phys. Lett. 486, 239 (2000).

68. T. Klähn, C. D. Roberts, L. Chang, H. Chen and Yu-Xin Liu, Phys. Rev. C 82, 035801 (2010).

69. A. Kurkela, P. Romatschke and A. Vuorinen, Phys. Rev. D 81, 105021 (2010).

70. H. A. Bethe, G. E. Brown and G. E. Brown, Nucl. Phys. A 467, 791 (1987).

71. E. Friedman, A. Gal, and C. J. Batty, Nucl. Phys. A 579, 578 (1994).

72. J. Mares, W. Friedman, A. Gal and B. K. Jennings, Nucl. Phys. A 594, 311 (1995).

73. R. Knorren, M. Prakash and P. J. Ellis, Phys. Rev. C 52, 3470 (1995).

74. M. Prakash, Manju Prakash, J. M. Lattimer and C. J. Pethick, Âstrophys. J. Lett. 390, L77 (1992).

75. J. R. Stone, P. A. M. Guichon, H. M. Matevosyan and A. W. Thomas, Nucl. Phys., 
A 792, 341 (2007).

76. A. Gezerlis and J. Carlson, Phys. Rev. C 81, 025803 (2010).

77. K. Hebeler and A. Schwenk, Phys. Rev. C 82, 014304 (2010).

78. K. Hebeler, J. M. Lattimer, C. J. Pethick and A. Schwenk, Phys. Rev. Lett. 105, 161102 (2010).

79. A. W. Steiner, J. M. Lattimer and E. F. Brown, ApJ 722, 33 (2010).

80. B. Kiziltan, A. Kottas and S. E. Thorsett, submitted to ApJ; arXiv:1011.4291 (2010). 